\section{Effect of Contact Precautions on Wait Time from Emergency Room Disposition to Inpatient Admission}

To the Editor-There is increasing pressure to initiate active surveillance programs that screen all patients requiring hospital admission for infection due to multidrug-resistant organisms (MDROs). In some states, legislative mandates require that all inpatients be screened for methicillin-resistant Staphylococcus aureus (MRSA). However, the strategy of active detection and isolation has a number of adverse unintended consequences, ${ }^{1}$ as well as raising ethical concerns. ${ }^{2}$ Patients under contact precautions have higher rates of anxiety, depression, and dissatisfaction with care; fewer provider visits; and increased preventable adverse drug events. ${ }^{3}$

Often, a lack of these single-patient rooms in many hospitals requires cohorting patients with the same MDRO infections into same-sex, multipatient rooms, thus increasing the complexity of patient flow. The process of creating singlepatient rooms for the purpose of isolation requires excessive intrahospital transfers, which compound the potential for reduced monitoring, missed treatments, and increased psychological stress. ${ }^{4}$ Thus, we suspected that active detection and isolation programs decrease hospital throughput, increase emergency department (ED) crowding, and prolong transfers from intensive care units (ICUs) to wards. According to the Society for Healthcare Epidemiology of America/Association for Professionals in Infection Control and Epidemiology position statement, it is estimated that $7.9 \%$ of all patients admitted would require contact precautions if an active surveillance program were mandated ${ }^{5}$ and institutions that implement active detection and isolation could expect to quadruple the number of patients requiring contact precautions. ${ }^{2}$ The objective of this study was to analyze ED disposition to inpatient admission wait times for patients with a history of infection or colonization with MDROs requiring contact precautions.

The study was performed in a 779-bed, urban, tertiary care, safety-net hospital with $38 \%$ of private beds in the non-ICU setting. A database of 6,255 admissions from ED to inpatient beds over a 4-month period (June 30-October 30, 2007) was reviewed. Patients that were admitted to pediatric services, that were sent directly to the operating room or for cardiac catheterization, or that were admitted to the ED for observation were excluded ( $n=2,149)$. Of the remaining 4,106 case patients, 1,500 were randomly selected for review. Length of stay from the time of the decision to admit to the time of ED discharge was calculated. Case patients were crossreferenced to the hospital isolation list of patients known to have a previous MDRO infection that would require contact precautions, as the hospital's policy is to isolate on admission those patients who at the time of prior discharge were isolated for MDRO colonization or infection. To avoid confounding, patients admitted to telemetry beds or stepdown beds ( $n=356)$ were excluded, since excessive wait times commonly occur as a result of limited availability of these beds. An additional 25 case patients were excluded from analysis because they required airborne or droplet precautions. ICU patients $(n=232)$ were also excluded from analysis because all ICU rooms are private and therefore the need for contact precautions would have no impact on wait time.

Of 825 non-ICU patients, $62(7.0 \%)$ required contact precautions on the basis of cultures that yielded MDROs during previous hospitalization. Mean wait time to admission was 298 minutes for the 62 patients requiring contact precautions, compared with 244 minutes for the 825 patients who did not (mean difference, 54 minutes; $P=.045$ ).

Without an active detection and isolation program, patients requiring contact precautions currently wait significantly longer (approximately 1 hour) for a ward bed than do those who do not require contact precautions. These patients are essentially competing for private rooms or must be cohorted with another patient colonized or infected with the same organism. If an active surveillance program were to be initiated, a significant increase in patients requiring contact precautions could be anticipated. At our institution, a 4-fold increase would equate to $28 \%$, meaning that approximately 1 out of every 4 patients admitted would require contact precautions. A similar study, analyzing effects of an MRSA active detection and isolation program on $\mathrm{ED}$ wait times at an academic medical center, concluded that patients who had been previously colonized or infected with MRSA waited 12.9 hours in the ED for a hospital bed, compared with 10.4 hours for non-MRSA patients (difference, 2.5 hours; $P=.001$ ). ${ }^{6}$ The authors suggested that there is an imbalance between national and local policies regarding isolation and overall general welfare of $\mathrm{ED}$ patients.

Prolonged ED wait times exacerbate problems associated with ED and hospital overcrowding. This leads to decreased hospital throughput and associated reductions in quality of care, such as prolonged time to administration of the first dose of antibiotics, prolonged time to administration of pain relief, and care that occurs in a less-than-optimal setting. ${ }^{2}$ With an active detection and isolation program, wait times can be expected to rise, with a concordant rise in the negative impact of isolation of patients who are colonized and not infected with MDROs.

\section{ACKNOWLEDGMENTS}

Potential conflicts of interest. All authors report no conflicts of interest relevant to this article. 
Aaron McLemore, $\mathrm{MD}^{1}$ Gonzalo Bearman, MD, MPH; Michael B. Edmond, MD, MPH, MPA ${ }^{1}$

Affiliations: 1. Division of Infectious Diseases, Department of Internal Medicine; Virginia Commonwealth University Medical Center, Richmond, Virginia.

Address reprint requests to Aaron McLemore, MD, Box 980019, Virginia Commonwealth University Medical Center, Richmond, VA 23298-0019 (admclemore@mcvh-vcu.edu).

Infect Control Hosp Epidemiol 2011;32(3):298-299

(C) 2011 by The Society for Healthcare Epidemiology of America. All rights reserved. 0899-823X/2011/3203-0017\$15.00. DOI: $10.1086 / 658913$

\section{REFERENCES}

1. Diekema DJ, Edmond MB. Look before you leap: active surveillance for multidrug-resistant organisms. Clin Infect Dis 2007; 44:1101-1107.

2. Edmond MB, Lyckholm L, Diekema DJ. Ethical implications of active surveillance cultures and contact precautions for controlling multidrug resistant organisms in the hospital setting. Public Health Ethics 2008;1:235-245.

3. Morgan DJ, Diekema DJ, Sepkowitz K, Perencevich EN. Adverse outcomes associated with contact precautions: a review of the literature. Am J Infect Control 2009;37:85-93.

4. Detsky ME, Etchells E. Single-patient rooms for safe patientcentered hospitals. JAMA 2008;300:954-956.

5. Weber SG, Huang SS, Oriola S, et al. Legislative mandates for use of active surveillance cultures to screen for methicillinresistant Staphylococcus aureus and vancomycin-resistant enterococci: position statement from the joint SHEA and APIC task force. Am J Infect Control 2007;35:73-85.

6. Gilligan P, Quirke M, Winder S, Humphreys H. Impact of admission screening for methicillin-resistant Staphylococcus aureus on the length of stay in an emergency department. $J$ Hosp Infect 2010;75:99-102.

\section{Clamping Down on Catheter-Related Bloodstream Infection}

To the Editor-At our institution, a large tertiary care hospital in Los Angeles, we have noted recurrent misuse of valved reflux-type intravenous catheter caps that may be contributing to increased rates of central line infections. Recently a 91-year-old male with an indwelling right femoral triplelumen central venous catheter developed Staphylococcus aureus bacteremia, as documented by 2 positive blood cultures, one of a sample drawn from the central line and another of a sample drawn from a peripheral site. The primary physician requested line removal. On arrival at the bedside, the dressing was minimally soiled, and the line site was without erythema, tenderness, or discharge. Valved positive-pressure flush caps (CLC-2000; ICU Medical) were present on all 3 lumens, as was standard practice at our institution until recently. Closer examination revealed all 3 ports to be clamped proximal to the hubs. All 3 caps were also noted to have depressed centers, consistent with the ports having been clamped before disconnecting them from the flush syringe (Figure 1). The line was removed without difficulty.

Since their introduction in the late 1990s, positive-pressure valved catheter caps have been introduced at many institutions to decrease needle-related injuries to staff, reduce catheter occlusion rates, and reduce the need for heparin flushing-all of which are important goals. ${ }^{1,2}$ Many institutions, however, have documented increased catheter-related bloodstream infection rates following the introduction of these devices. $^{3-8}$

Connection of a Luer lock access device to the CLC-2000 cap compresses a spring-loaded piston within the cap. When the access device is disconnected, this spring moves the piston outward to its baseline position. As the piston moves outward, it provides a positive-pressure flush through the catheter lumen. Clamping the catheter proximal to the hub prevents this flushing action, causing the piston or plunger to remain depressed below the surface of the cap housing. With the piston in the depressed position, the interior surface of the cylindrical cap body is exposed to air, and the piston surface is several millimeters below the surface of the cylindrical body. A 70\% isopropyl alcohol swab cannot contact the surface of the piston in this position and cannot reach the interior of the cap body. With the piston depressed, it is impossible to disinfect the cap adequately with conventional nursing practice methods, potentially leaving nondisinfected surfaces exposed to infusate when the cap is next connected to a Luer lock device.

Recommended clamping procedures for valved positivepressure caps differ from other types of cap. Needle-based access devices, for example, require catheter clamping prior to removal of the access needle to prevent blood reflux into the catheter tip. Many needleless split-septum (ie, non-positive pressure) devices require clamping prior to access device removal also, for similar reasons. Positive-displacement mechanical valve caps, however, require just the opposite sequence: de-access, then clamp. In the busy world of patient care, the distinction between a positive-displacement cap and a non-positive-displacement cap is easily overlooked, leading to suboptimal access and de-access procedures. Unfortunately, the implication of this simple difference between cap types is potentially serious: with a valved device, the improper access sequence not only prevents it from flushing as designed but is likely to also prevent adequate surface decontamination when the device is next accessed.

Positive-displacement valved catheter caps previously have been linked to increased catheter-related infection rates in intensive care settings, ${ }^{6}$ long-term care institutions, ${ }^{8}$ general inpatient settings, ${ }^{3}$ and hematology-oncology wards. ${ }^{4,7}$ Though these caps have been shown by culture to be contaminated, ${ }^{3}$ to our knowledge, no obvious mechanism of contamination related to use of these devices has yet been suggested in the literature. While we cannot, on the basis of our 